\title{
Bounds for test exponents
}

\author{
Holger Brenner
}

\begin{abstract}
Suppose that $R$ is a two-dimensional normal standard-graded domain over a finite field. We prove that there exists a uniform Frobenius test exponent $b$ for the class of homogeneous ideals in $R$ generated by at most $n$ elements. This means that for every ideal $I$ in this class we have that $f^{p^{b}} \in I^{\left[p^{b}\right]}$ if and only if $f \in I^{\mathrm{F}}$. This gives in particular a finite test for the Frobenius closure. On the other hand we show that there is no uniform bound for Frobenius test exponent for all homogeneous ideals independent of the number of generators. Under similar assumptions we prove also the existence of a bound for tight closure test ideal exponents for ideals generated by at most $n$ elements.
\end{abstract}

\section{Introduction}

In this paper we deal with test exponents for Frobenius closure and for tight closure. In this introduction we will concentrate on the Frobenius closure and we will come back to tight closure in $\S 6$. Suppose that $R$ denotes a commutative noetherian ring containing a field of positive characteristic $p$, and let $I \subseteq R$ denote an ideal. The Frobenius closure, written $I^{\mathrm{F}}$, is the ideal

$$
I^{\mathrm{F}}=\left\{f \in R: \exists e \in \mathbb{N} \text { such that } f^{p^{e}} \in I^{\left[p^{e}\right]}\right\} .
$$

Here the Frobenius power $I^{[q]}=\left(f^{q}: f \in I\right)$ is the extended ideal of $I$ under the eth iteration of the Frobenius homomorphism $R \rightarrow R, f \mapsto f^{q}$, where $q=p^{e}$. Since $I^{\mathrm{F}}$ is an ideal in a noetherian ring, there exists a number $b$ such that $f^{p^{b}} \in I^{\left[p^{b}\right]}$ for every $f \in I^{\mathrm{F}}$.

A problem of Katzman and Sharp [KS04, Introduction] asks in its strongest form: does there exist a number $b$, depending only on the ring $R$, such that, for every ideal and for every $f \in I^{\mathrm{F}}$, we have $f^{p^{b}} \in I^{\left[p^{b}\right]}$ ? A positive answer to this question, together with the actual knowledge of a bound for $b$, would give an algorithm to compute the Frobenius closure $I^{\mathrm{F}}$. We call such a number $b$ a Frobenius test exponent for the ring $R$.

A weaker question is this: for a given ideal $I$, does there exist a Frobenius test exponent for all Frobenius powers $I^{[q]}$ ? The existence of such a weak Frobenius test exponent $b$ means that $f \in\left(I^{[q]}\right)^{\mathrm{F}}$ holds if and only if $f^{p^{b}} \in\left(I^{[q]}\right)^{\left[p^{b}\right]}$. Katzman and Sharp show that for an ideal generated by a regular sequence there exists such a weak Frobenius test exponent.

An intermediate question is the following: does there exist a Frobenius test exponent for ideals generated by at most a fixed number of elements? Since $\left(f_{1}, \ldots, f_{n}\right)^{[q]}=\left(f_{1}^{q}, \ldots, f_{n}^{q}\right)$, the Frobenius powers of an ideal generated by $n$ elements do not need more generators than $n$; therefore a positive answer to this question implies a positive answer to the weak question of Katzman and Sharp. Moreover, a positive answer to this question has the same computational impact on the decision whether $f \in I^{\mathrm{F}}$ holds or not.

Received 26 December 2004, accepted in final form 22 June 2005.

2000 Mathematics Subject Classification 13A35, 14D20, 14F05, 14H52, $14 \mathrm{H} 60$.

Keywords: Frobenius closure, tight closure, test exponent, semistable bundles.

This journal is (C) Foundation Compositio Mathematica 2006. 


\section{H. BRENNER}

In this paper we consider the case of homogeneous $R_{+}$-primary ideals $I$ in a geometrically normal two-dimensional standard-graded domain $R$ over a field $\mathbb{F}$ of positive characteristic. We obtain the following results. If $R$ is the homogeneous coordinate ring of an elliptic curve, then the answer to the third question is affirmative. There is a Frobenius test exponent $b$ for the class of homogeneous $R_{+}$-primary ideals generated by at most $n$ elements, and in fact one can take $b=n-1$ (Theorem 1.1). This is an application of the Atiyah classification of vector bundles over an elliptic curve.

In Theorem 2.4 we give a negative answer to the strong question of Katzman and Sharp. We show that for a homogeneous coordinate ring $R$ over an elliptic curve with Hasse-invariant 0 there does not exist a uniform Frobenius test exponent valid for all homogeneous ideals in $R$. This relies on results of Oda about properties of the Frobenius pull-backs of the bundles $F_{r}$ on an elliptic curve, where $F_{r}$ denotes the unique indecomposable bundle of rank $r$ and degree 0 with a non-trivial section, and on realizing $F_{r}$ as a syzygy bundle for some ideal generators.

For homogeneous normal coordinate rings of curves of higher genus, we obtain in Theorem $5.1 \mathrm{a}$ positive answer to the third question under the condition that the base field is finite. This is deduced from the geometric interpretation of tight closure in terms of vector bundles on the corresponding smooth projective curve and the boundedness of semistable bundles on the curve of given rank and degree. The boundedness implies that there is a finite test for strong semistability if everything is defined over a finite field (Lemma 3.1). From this we deduce using a recent result of Trivedi that there is also a finite algorithm to find the strong Harder-Narasimhan filtration of a bundle of given rank (Corollary 3.2).

These two results are important ingredients in an argument to show that a cohomology class $c \in H^{1}(C, \mathcal{S})$ of a locally free sheaf of rank $r$ on a smooth projective curve defined over a finite field which is annihilated by some Frobenius power is in fact annihilated by a certain fixed power which depends only on the rank and on the curve, but not on the sheaf itself (Theorem 4.2). This yields eventually the Frobenius test exponent for ideals generated by at most $n$ elements (Theorem 5.1). However, this Frobenius test exponent is (even for ideals generated by three elements in the coordinate ring over a curve of genus 2) hardly a basis for implementing an algorithm to compute the Frobenius closure.

In the same spirit we prove in Theorem 6.4 the existence of a uniform test ideal exponent for ideals generated by at most $n$ elements, under the assumption that $R$ is a two-dimensional standard-graded geometrically normal Gorenstein domain over a finite field.

\section{Frobenius test exponents over elliptic curves}

The vector bundles (locally free sheaves) on an elliptic curve $C$ over an algebraically closed field are well understood due to the classification of Atiyah; see [Ati57]. We recall briefly some consequences of this classification. If $\mathcal{S}$ is an indecomposable bundle, meaning that there is no non-trivial decomposition $\mathcal{S}=\mathcal{F} \oplus \mathcal{G}$ with vector bundles $\mathcal{F}$ and $\mathcal{G}$ of smaller ranks, then the following hold: If $\operatorname{deg}(\mathcal{S})>0$, then $H^{1}(C, \mathcal{S})=0$. If $\operatorname{deg}(\mathcal{S})=0$, then either $H^{0}(C, \mathcal{S})=H^{1}(C, \mathcal{S})=0$ or $\mathcal{S}=F_{r}$, the unique indecomposable sheaf of rank $r$ and degree 0 with a non-trivial global section. These sheaves have $\operatorname{dim} H^{0}\left(C, F_{r}\right)=\operatorname{dim} H^{1}\left(C, F_{r}\right)=1$, they are self-dual and they are related by the non-trivial extensions $0 \rightarrow F_{r-1} \rightarrow F_{r} \rightarrow \mathcal{O}_{C} \rightarrow 0$.

Theorem 1.1. Let $R$ denote a normal standard-graded two-dimensional domain over a field $\mathbb{F}$ of positive characteristic $p$ and suppose that $C=\operatorname{Proj} R$ is an elliptic curve. Let $I=\left(f_{1}, \ldots, f_{n}\right)$ denote an $R_{+}$-primary ideal generated by $n$ homogeneous elements. Suppose that the element $f$ belongs to the Frobenius closure of $I$. Then already $f^{q} \in I^{[q]}$ holds for $q=p^{n-1}$. Hence $n-1$ is a uniform Frobenius test exponent for all homogeneous $R_{+}$-primary ideals generated by at most $n$ elements. 


\section{BOUNDS FOR TEST EXPONENTS}

Proof. We may assume that $\mathbb{F}$ is algebraically closed. Since $I$ is homogeneous, all Frobenius powers $I^{[q]}$ and the Frobenius closure $I^{\mathrm{F}}$ are also homogeneous. Therefore we may assume that $f$ is homogeneous. A homogeneous element $f$ of degree $m$ yields via the short exact sequence

$$
0 \longrightarrow \operatorname{Syz}\left(f_{1}, \ldots, f_{n}\right)(m) \longrightarrow \bigoplus_{i=1}^{n} \mathcal{O}_{C}\left(m-d_{i}\right) \stackrel{f_{1}, \ldots, f_{n}}{\longrightarrow} \mathcal{O}_{C}(m) \longrightarrow 0
$$

of locally free sheaves on $C$ the class $\delta(f) \in H^{1}\left(C, \operatorname{Syz}\left(f_{1}, \ldots, f_{n}\right)(m)\right)$. The pull-back of this sequence under the eth absolute Frobenius morphism $\Phi^{e}: C \rightarrow C$ is $\left(q=p^{e}\right)$

$$
0 \longrightarrow \Phi^{e *}\left(\operatorname{Syz}\left(f_{1}, \ldots, f_{n}\right)(m)\right) \longrightarrow \bigoplus_{i=1}^{n} \mathcal{O}_{C}\left(q m-q d_{i}\right) \stackrel{f_{1}^{q}, \ldots, f_{n}^{q}}{\longrightarrow} \mathcal{O}_{C}(q m) \longrightarrow 0
$$

therefore $\operatorname{Syz}\left(f_{1}^{q}, \ldots, f_{n}^{q}\right)(q m)=\Phi^{e *}\left(\operatorname{Syz}\left(f_{1}, \ldots, f_{n}\right)(m)\right)$. From this we see that $f^{q} \in I^{[q]}=$ $\left(f_{1}^{q}, \ldots, f_{n}^{q}\right)$ if and only if the $e$ th Frobenius pull-back of the class $\delta(f)$ is zero, that is $\Phi^{e *}(\delta(f))=0$ in $H^{1}\left(C, \operatorname{Syz}\left(f_{1}^{q}, \ldots, f_{n}^{q}\right)(q m)\right)$. The element $f$ belongs to the Frobenius closure of $I=\left(f_{1}, \ldots, f_{n}\right)$ if and only if some Frobenius power of this cohomology class vanishes.

Let $\operatorname{Syz}\left(f_{1}, \ldots, f_{n}\right)(m)=\mathcal{S}_{1} \oplus \cdots \oplus \mathcal{S}_{k}$ denote the decomposition of the syzygy bundle into indecomposable bundles, and denote the components of the cohomology class $c=\delta(f)$ by $c_{j} \in$ $H^{1}\left(C, \mathcal{S}_{j}\right)$. For the $\mathcal{S}_{j}$ of positive degree we have $H^{1}\left(C, \mathcal{S}_{j}\right)=0$. If $\mathcal{S}_{j}$ has negative degree and $c_{j} \neq 0$, then $f$ does not belong to the tight closure of the ideal by [Bre05, Corollary 4.1] and so it does not belong to the Frobenius closure. So these components are also zero. For an indecomposable sheaf $\mathcal{S}$ of degree 0 we have two possibilities depending on whether $\mathcal{S}$ has no non-trivial global sections or it has. In the first case we have again $H^{1}(C, \mathcal{S})=0$. In the second case we have $\mathcal{S}=F_{r}$, the unique indecomposable sheaf of rank $r$ and degree 0 with a non-trivial global section. Furthermore $r \leqslant n-1=\operatorname{rk}\left(\operatorname{Syz}\left(f_{1}, \ldots, f_{n}\right)(m)\right)$.

The indecomposable bundles $F_{r}$ are related by short exact sequences

$$
0 \longrightarrow F_{r-1} \longrightarrow F_{r} \longrightarrow \mathcal{O}_{C} \longrightarrow 0,
$$

where $1 \in \mathbb{F}=\Gamma\left(C, \mathcal{O}_{C}\right)$ maps to a non-trivial element in the one-dimensional space $H^{1}\left(C, F_{r-1}\right)$. Suppose first that the Hasse-invariant of the elliptic curve is 0, so that the Frobenius morphism $\Phi^{*}: H^{1}\left(C, \mathcal{O}_{C}\right) \rightarrow H^{1}\left(C, \mathcal{O}_{C}\right)$ is the zero map. In this case we show by induction on $r$ that the $(r-1)$ th Frobenius pull-back trivializes $F_{r}$ (meaning that $\Phi^{(r-1) *}\left(F_{r}\right) \cong \mathcal{O}_{C}^{r}$ ) and that the $r$ th Frobenius pull-back annihilates $H^{1}\left(C, F_{r}\right)$. For $F_{1}=\mathcal{O}_{C}$ this follows from the assumption that the Hasse-invariant is 0 . Now suppose that $r \geqslant 2$. The $(r-2)$ th Frobenius pull-back of $0 \rightarrow F_{r-1} \rightarrow F_{r} \rightarrow \mathcal{O}_{C} \rightarrow 0$ yields an exact sequence

$$
0 \longrightarrow \mathcal{O}_{C}^{r-1} \longrightarrow \Phi^{(r-2) *}\left(F_{r}\right) \longrightarrow \mathcal{O}_{C} \longrightarrow 0 .
$$

This extension is given by a cohomology class in $H^{1}\left(C, \mathcal{O}_{C}^{r-1}\right)$, and so application of the Frobenius once more shows that the extension is trivial; hence $\Phi^{(r-1) *}\left(F_{r}\right) \cong \mathcal{O}_{C}^{r}$. Another application of the Frobenius then annihilates the cohomology coming from $F_{r}$.

If the Hasse-invariant of $C$ is 1 , then the Frobenius acts bijectively on $H^{1}\left(C, \mathcal{O}_{C}\right)$. The short exact sequences relating $F_{r-1}$ and $F_{r}$ induce isomorphisms $H^{1}\left(C, F_{r}\right) \cong H^{1}\left(C, \mathcal{O}_{C}\right)$. Hence by induction on $r$ we get that $\Phi^{*}\left(F_{r}\right) \cong F_{r}$ and that $\Phi^{*}: H^{1}\left(C, F_{r}\right) \rightarrow H^{1}\left(C, \Phi^{*}\left(F_{r}\right)\right)$ is a bijection. Hence the Frobenius does not annihilate anything.

Remark 1.2. Theorem 1.1 implies that the Frobenius closure of a homogeneous $R_{+}$-primary ideal in a normal homogeneous coordinate ring $R$ of an elliptic curve can be computed by an easy algorithm. The same argument shows (for Hasse-invariant 0 or 1 ) that an element $f$ belongs to the plus closure $I^{+}$(or to the tight closure) of $I$ if and only if it belongs to the extended ideal under the $(n-1)$ th iteration of the ring homomorphism $\varphi: R \rightarrow R$ which describes the $p$-multiplication 


\section{H. BRENNER}

of the elliptic curve. This shows that the tight closure of an $R_{+}$-primary homogeneous ideal in $R$ is also algorithmically computable. Another easy algorithm for the computation of tight closure in $R=\mathbb{F}[x, y, z] /(G)$, where $G$ is a cubic polynomial, in terms of test ideal exponent is given in Corollary 6.6 below.

\section{Unboundedness of Frobenius test exponents}

In this section we show that in a homogeneous coordinate ring $R$ of an elliptic curve with Hasseinvariant 0 there does not exist a bound for Frobenius test exponents which holds uniformly for all homogeneous ideals.

We recall the following result of Oda about the Frobenius pull-backs of the bundles $F_{r}$ on an elliptic curve and how the Frobenius acts on the cohomology [Oda71, Proposition 2.10 and Theorem 2.17].

Theorem 2.1. Let $C$ denote an elliptic curve over a field $\mathbb{F}$ of positive characteristic $p$ and let $F_{r}$ denote the unique indecomposable bundle on $C$ of rank $r$ and degree 0 with $H^{0}\left(C, F_{r}\right)=\mathbb{F} \neq 0$. Let $\Phi: C \rightarrow C$ denote the Frobenius. Then the following hold.

(i) If the Hasse-invariant of $C$ is non-zero, then $\Phi^{*}\left(F_{r}\right) \cong F_{r}$.

(ii) If the Hasse-invariant of $C$ is zero, then

$$
\Phi^{*}\left(F_{r}\right) \cong \mathcal{O}_{C}^{r} \quad \text { for } r \leqslant p \quad \text { and } \quad \Phi^{*}\left(F_{r}\right) \cong \bigoplus_{i=1}^{p} F_{\lfloor(r-i) / p\rfloor+1} \quad \text { for } r>p .
$$

(iii) The map $\Phi^{*}: H^{1}\left(C, F_{r}\right) \rightarrow H^{1}\left(C, \Phi^{*}\left(F_{r}\right)\right)$ is injective if and only if the Hasse-invariant is non-zero or $r \geqslant p$ (and for Hasse-invariant zero and $r<p$ it is the zero map).

From this result of Oda we deduce the corollary that there does not exist a uniform bound for Frobenius test exponents for all locally free sheaves on $C$ independent of the rank.

Corollary 2.2. Let $C$ denote an elliptic curve over a field $\mathbb{F}$ of positive characteristic $p$ and suppose that the Hasse-invariant is 0 . Then for every number $b \in \mathbb{N}$ there exists a locally free sheaf $\mathcal{S}$ on $C$ and a cohomology class $c \in H^{1}(C, \mathcal{S})$ such that $c$ is annihilated by some Frobenius power, but such that $\Phi^{b *}(c) \neq 0$.

Proof. We show that for $0 \neq c \in H^{1}\left(C, F_{r}\right)$, where $r \geqslant p^{b+1}$, we have that $\Phi^{b *}(c) \in H^{1}\left(C, \Phi^{b *}\left(F_{r}\right)\right)$ is not zero, though it is annihilated by some Frobenius power, since the curve is assumed to have Hasse-invariant 0 (see the proof of Theorem 1.1). We use induction on $b$, the case where $b=0$ being clear. So suppose that $b \geqslant 1$. By Theorem 2.1(ii) and (iii) we have the decomposition

$$
\Phi^{*}\left(F_{r}\right) \cong \bigoplus_{i=1}^{p} F_{\lfloor(r-i) / p\rfloor+1}
$$

and we know that the mapping on the cohomology is injective. Hence at least one component of $\Phi^{*}(c)$ is non-zero, say $c^{\prime}=\left(\Phi^{*}(c)\right)_{i} \in H^{1}\left(C, F_{\lfloor(r-i) / p\rfloor+1}\right)$. The rank of $F_{\lfloor(r-i) / p\rfloor+1}$ is at least

$$
\left\lfloor\frac{r-i}{p}\right\rfloor+1 \geqslant\left\lfloor\frac{r-p}{p}\right\rfloor+1=\left\lfloor\frac{r}{p}\right\rfloor \geqslant\left\lfloor\frac{p^{b+1}}{p}\right\rfloor=p^{b} .
$$

By the induction hypothesis we know that $\Phi^{(b-1) *}\left(c^{\prime}\right) \neq 0$; hence $\Phi^{b *}(c)=\Phi^{(b-1) *}\left(\Phi^{*}(c)\right) \neq 0$.

We are going to translate these results into results about the Frobenius closure of ideals. To do so we have to realize the bundles $F_{r}$ on an elliptic curve as a syzygy bundle for suitable ideal generators. The following lemma is known. 


\section{BOUNDS FOR TEST EXPONENTS}

Lemma 2.3. Let $C$ denote a smooth projective curve over an algebraically closed field, and let $\mathcal{S}$ denote a locally free sheaf of rank $r$, which is globally generated by $r+k$ global sections, $k \geqslant 1$. Then it is also globally generated by $r+1$ global sections.

Proof. The assumption means that we have a surjection

$$
\mathcal{O}_{C}^{r+k} \longrightarrow \mathcal{S} \longrightarrow 0 \text {. }
$$

Set $V=\Gamma\left(C, \mathcal{O}_{C}^{r+k}\right)$, and consider the family of subspaces $U \subset V$ of codimension one, which form an $(r+k)$-dimensional Grassmann variety $G(r+k-1, V)$. Fix $x \in C$ and consider the surjection

$$
\varphi_{x}: V \longrightarrow W:=\mathcal{S} \otimes \kappa(x) .
$$

Let $K=K_{x}$ denote its kernel. The intersection of $U$ with $K$ has either dimension $k-1$ or it is $K$. In the first case $U$ maps onto $W$, in the second case not. The subspaces $U$ of dimension $r+k-1$ which contain $K$ are given by the $(r-1)$-dimensional subspaces of $V / K$, so they form an $r$-dimensional subvariety $G_{x}$ of $G(r+k-1, V)$. Hence $\bigcup_{x \in C} G_{x}$ has dimension at most $r+1$, and so for $k \geqslant 2$ the generic hyperplane $U \subset V$ also generates $\mathcal{S}$ globally. So inductively we can reduce $k$ until we have only $r+1$ generators.

Theorem 2.4. Let $R$ denote a normal homogeneous coordinate ring over an elliptic curve of positive characteristic $p$ and of Hasse-invariant 0. Then there does not exist a Frobenius test exponent valid for all homogeneous $R_{+}$-primary ideals $I \subseteq R$.

Proof. Let $C=\operatorname{Proj} R$ denote the corresponding elliptic curve and let $\mathcal{O}_{C}(1)$ denote the ample invertible sheaf on $C$. For some $\ell>0$ the twisted bundle $F_{r}(\ell)$ on $C$ is globally generated. Owing to Lemma 2.3 there exists a short exact sequence

$$
0 \longrightarrow \mathcal{O}_{C}(-d) \longrightarrow \mathcal{O}_{C}^{r+1} \longrightarrow F_{r}(\ell) \longrightarrow 0 \quad(d>0)
$$

Dualizing and tensoring with $\mathcal{O}(\ell)$ yields (since $F_{r}^{\vee}=F_{r}$ )

$$
0 \longrightarrow F_{r} \longrightarrow \mathcal{O}_{C}^{r+1}(\ell) \longrightarrow \mathcal{O}_{C}(d+\ell) \longrightarrow 0
$$

where the last mapping is given by some homogeneous elements $f_{1}, \ldots, f_{r+1}$ of degree $d$, hence $F_{r} \cong \operatorname{Syz}\left(f_{1}, \ldots, f_{r+1}\right)(d+\ell)$. Since $\ell>0$, we have $H^{1}(C, \mathcal{O}(\ell))=0$ and therefore the non-trivial class $c \in H^{1}\left(C, F_{r}\right)$ comes from an element $f \in \Gamma\left(C, \mathcal{O}_{C}(d+\ell)\right)=R_{d+\ell}$. By Corollary 2.2 and its proof there exists for given $b$ a number $r$ such that $0 \neq c \in H^{1}\left(C, F_{r}\right)$ is not annihilated by the $b$ th Frobenius power, but it is annihilated by some Frobenius power. This means that $f$ belongs to the Frobenius closure of the ideal $I=\left(f_{1}, \ldots, f_{r+1}\right)$, but $f^{p^{b}} \notin I^{\left[p^{b}\right]}$.

\section{Semistable sheaves on curves over a finite field}

We look now at normal homogeneous coordinate rings over a smooth projective curve $C$ of higher genus. The theory of vector bundles on $C$ is still the main ingredient in the following results, but since this theory is more complicated than in the elliptic case we obtain our results only under the condition that everything is defined over a finite field.

Recall some facts (see [HL97] or [Ses82] for details) about locally free sheaves on a smooth projective curve $C$ over a field $\mathbb{F}$. The degree of a locally free sheaf $\mathcal{S}$ on $C$ of rank $r$ is defined by $\operatorname{deg}(\mathcal{S})=\operatorname{deg} \bigwedge^{r}(\mathcal{S})$; the degree is additive on short exact sequences. The slope of $\mathcal{S}$, written $\mu(\mathcal{S})$, is defined to be $\operatorname{deg}(\mathcal{S}) / r$. The slope has the property that $\mu(\mathcal{S} \otimes \mathcal{T})=\mu(\mathcal{S})+\mu(\mathcal{T})$.

A locally free sheaf $\mathcal{S}$ is called semistable (respectively stable) if $\mu(\mathcal{T}) \leqslant \mu(\mathcal{S})$ (respectively $\mu(\mathcal{T})<\mu(\mathcal{S})$ ) for every locally free subsheaf $\mathcal{T} \subset \mathcal{S}$. Tensoring with an invertible sheaf does not 


\section{H. BRENNER}

affect this property. A non-zero morphism $\mathcal{S} \rightarrow \mathcal{T}$ between two semistable sheaves enforces $\mu(\mathcal{T}) \geqslant \mu(\mathcal{S})$. In particular, a semistable sheaf of negative degree does not have any non-trivial global section.

For fixed rank $r$ and degree $d$ the set of semistable sheaves form a bounded family: see [HL97, 1.7] or [Ses82, III.A]. This is a basic result in the construction of the moduli space of (semi)stable sheaves on a curve and on higher-dimensional varieties. This boundedness implies in particular that there are only finitely many semistable sheaves of rank $r$ and of degree $d$ defined over a finite field.

In positive characteristic $p$, a locally free sheaf $\mathcal{S}$ is called strongly semistable if every Frobenius pull-back $\Phi^{e *}(\mathcal{S})$ under the eth absolute Frobenius $\Phi: C \rightarrow C$ is again semistable. The following lemma shows that, if everything is defined over a finite field, then we only have to look at certain Frobenius powers to test for strong semistability.

Lemma 3.1. Let $C$ denote a smooth projective curve defined over a finite field $\mathbb{F}$, and let $r \in \mathbb{N}$. Then there exists a number $m$ such that for every locally free sheaf $\mathcal{S}$ of rank $r$ on $C$ the following holds: if $\Phi^{m *}(\mathcal{S})$ is semistable, then $\mathcal{S}$ is strongly semistable.

Proof. By enlarging the ground field we may assume that there exists an $\mathbb{F}$-rational point on $C$ and hence an invertible sheaf $\mathcal{L}$ of degree 1 . Let $\mathcal{S}$ be given of rank $r$. There exists $a \geqslant 0$ and $e>0$, $a+e \leqslant r$, such that $p^{a+e} \operatorname{deg}(\mathcal{S})=p^{a} \operatorname{deg}(\mathcal{S}) \bmod r$. Let $\mathcal{T}=\Phi^{a *}(\mathcal{S}) \otimes \mathcal{L}^{\ell}$, where $\ell$ is chosen such that $\delta:=\operatorname{deg}(\mathcal{T})=p^{a} \operatorname{deg}(\mathcal{S})+\ell r$ is in between 0 and $r-1$. There exists $k$ such that $p^{e} \delta-\delta=k r$. Hence the assignment (and its iterations)

$$
\mathcal{T} \longmapsto \Phi^{e *}(\mathcal{T}) \otimes \mathcal{L}^{-k}=: \varphi(\mathcal{T})
$$

preserves the degree, since $\operatorname{deg}\left(\Phi^{e *}(\mathcal{T}) \otimes \mathcal{L}^{-k}\right)=p^{e} \operatorname{deg}(\mathcal{T})-k r$. The number of semistable bundles defined over $\mathbb{F}$ of fixed rank $r$ and degree $\delta$ is finite, say bounded by $n$. Set $m=r(n+1)$.

If now $\Phi^{m *}(\mathcal{S})$ is semistable, then all $\Phi^{i *}(\mathcal{S})$ are semistable for $i \leqslant m$, but then of course the $\varphi^{j}(\mathcal{T})$ are semistable for all $j \leqslant n$. Since they have the same degree $\delta$, two of them must be isomorphic. But then we must have a periodicity among the $\varphi^{j}(\mathcal{T})$ and so they are in fact semistable for all $j \in \mathbb{N}$. Hence $\mathcal{S}$ is strongly semistable (see also [LS77] for this argument).

Note that we have to deal only with the finitely many degrees between 0 and $r-1$, so that there exists a bound which is independent of the degree of the bundles.

For every locally free sheaf $\mathcal{S}$ on $C$ there exists the so-called Harder-Narasimhan filtration $0 \subset \mathcal{S}_{1} \subset \cdots \subset \mathcal{S}_{t}=\mathcal{S}$, where the $\mathcal{S}_{j}$ are locally free subsheaves. This filtration is unique and has the property that the quotients $\mathcal{S}_{j} / \mathcal{S}_{j-1}$ are semistable and $\mu\left(\mathcal{S}_{j} / \mathcal{S}_{j-1}\right)>\mu\left(\mathcal{S}_{j+1} / \mathcal{S}_{j}\right)$ for all $j=1, \ldots, t-1$. In positive characteristic, a Harder-Narasimhan filtration is called strong if all quotients $\mathcal{S}_{j} / \mathcal{S}_{j-1}$ are strongly semistable. For every locally free sheaf $\mathcal{S}$ there exists a pull-back $\Phi^{e *}(\mathcal{S})$ such that its Harder-Narasimhan filtration is strong [Lan04, Theorem 2.7]. An observation of Trivedi combined with Lemma 3.1 allows us to give a bound for the Frobenius power such that the Harder-Narasimhan filtration is strong.

Corollary 3.2. Let $C$ denote a smooth projective curve of genus $g$ defined over a finite field $\mathbb{F}$, and let $r \in \mathbb{N}$. Suppose that $\operatorname{char}(\mathbb{F})=p>4(g-1) r^{3}$. Then there exists a number $h$ such that for every locally free sheaf $\mathcal{S}$ of rank $r$ the Harder-Narasimhan filtration of $\Phi^{h *}(\mathcal{S})$ is strong.

Proof. We use induction on $r$; for $r=1$ there is nothing to show. Set $h=(r-1) m$, where $m$ is the number for which the conclusion of Lemma 3.1 holds for all locally free sheaves of rank at most $r$. Consider $\Phi^{m *}(\mathcal{S})$. If this is semistable, then $\mathcal{S}$ is strongly semistable by Lemma 3.1 and we have the desired result. So suppose that

$$
0 \subset \mathcal{S}_{1} \subset \cdots \subset \mathcal{S}_{t-1} \subset \mathcal{S}_{t}=\Phi^{m *}(\mathcal{S})
$$




\section{BOUNDS FOR TEST EXPONENTS}

is the Harder-Narasimhan filtration, with $\operatorname{rk}\left(\mathcal{S}_{j} / \mathcal{S}_{j-1}\right)<r$. By the theorem of Trivedi [Tri04] (here the condition about the prime characteristic is needed) the Harder-Narasimhan filtration of a higher Frobenius pull-back is a refinement of the pull-back of this filtration. Hence we can apply the induction hypothesis to the quotient sheaves $\mathcal{S}_{j} / \mathcal{S}_{j-1}$.

Remark 3.3. For $r=2$ we do not need the condition about the prime characteristic, since then $\Phi^{m}(\mathcal{S})$ is either semistable ( $m$ being the invariant from Lemma 3.1), and hence strongly semistable, or it has an invertible subsheaf which contradicts semistability. In any case the Harder-Narasimhan filtration of $\Phi^{m}(\mathcal{S})$ is strong.

\section{Frobenius test exponents for curves of higher genus}

Let $C$ denote a smooth projective curve over a finite field and let $\mathcal{S}$ denote a locally free sheaf on $C$. We say that a cohomology class $c \in H^{1}(C, \mathcal{S})$ is annihilated by some Frobenius if $\Phi^{e *}(c) \in$ $H^{1}\left(C, \Phi^{e *}(\mathcal{S})\right)$ is zero for some $e \in \mathbb{N}$. We want to show that this can be checked within a certain number of steps, which is bounded by a number only depending on $C$ and on the rank of $\mathcal{S}$. We restrict first to strongly semistable sheaves.

Lemma 4.1. Let $C$ denote a smooth projective curve defined over a finite field $\mathbb{F}$, and let $r \in \mathbb{N}$. Then there exists a number $k$ such that for every strongly semistable locally free sheaf $\mathcal{T}$ on $C$ of rank $r$ the Frobenius annihilation of a cohomology class $c \in H^{1}(C, \mathcal{T})$ can be checked within $k$ steps.

Proof. We examine three cases depending on whether $\operatorname{deg}(\mathcal{T})$ is positive, zero or negative. In each case we will find a bound $k$.

Case 1. Suppose first that $\operatorname{deg}(\mathcal{T})>0$. Let $k$ be such that $p^{k}>r \operatorname{deg}\left(\omega_{C}\right)$, where $\omega_{C}$ denotes the dualizing sheaf on $C$; thus $\operatorname{deg}\left(\omega_{C}\right)=2 g-2$, where $g$ is the genus of the curve. Then $\Phi^{k *}(c) \in$ $H^{1}\left(C, \Phi^{k *}(\mathcal{T})\right)$ and the degree of this bundle is

$$
\operatorname{deg}\left(\Phi^{k *}(\mathcal{T})\right)=p^{k} \operatorname{deg}(\mathcal{T}) \geqslant p^{k} .
$$

Therefore $\mu\left(\Phi^{k *}(\mathcal{T})\right) \geqslant p^{k} / r>\operatorname{deg}\left(\omega_{C}\right)$. Hence there exists no non-trivial homomorphism $\Phi^{k *}(\mathcal{T}) \rightarrow \omega_{C}$ (due to strong semistability) and therefore $H^{1}\left(C, \Phi^{k *}(\mathcal{T})\right)=0$ by Serre duality. So the $k$ th Frobenius annihilates these classes.

Case 2. Suppose now that $\operatorname{deg}(\mathcal{T})=0$. The curve and $\mathcal{T}$ are defined over the finite field $\mathbb{F}$. The pull-back of $\mathcal{T}$ is again semistable of degree 0 and defined over $\mathbb{F}$. The number of semistable bundles of rank $r$ and degree 0 defined over a finite field is however finite. Call this number $n$, as in Lemma 3.1. Hence there must be a repetition, say $\Phi^{t *}(\mathcal{T}) \cong \Phi^{t^{\prime} *}(\mathcal{T})$, where $0 \leqslant t<t^{\prime} \leqslant n$. Set $t^{\prime}-t=: v$ and define $\mathcal{F}:=\Phi^{t *}(\mathcal{T})$.

Let $\theta: \Phi^{v *}(\mathcal{F}) \rightarrow \mathcal{F}$ be a fixed isomorphism. This induces isomorphisms $\Phi^{(k-1) v *}(\theta): \Phi^{k v *}(\mathcal{F}) \rightarrow$ $\Phi^{(k-1) v *}(\mathcal{F})$ and the composition of these yields isomorphisms $\theta_{k}: \Phi^{k v *}(\mathcal{F}) \rightarrow \mathcal{F}$. Set $\varphi=\theta \circ \Phi^{v *}:$ $\mathcal{F} \rightarrow \mathcal{F}$. The diagram

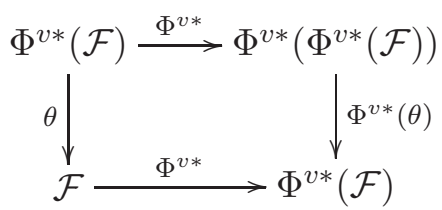

commutes by the functoriality of $\Phi^{*}$. Therefore $\theta_{k} \circ \Phi^{k v *}=\varphi^{k}$. Since the $\theta_{k}$ are isomorphisms, they induce isomorphisms on the cohomology groups, and so a cohomology class $c \in H^{1}(C, \mathcal{F})$ is annihilated by $\Phi^{k v *}$ if and only if it is annihilated by $\varphi^{k}$. 


\section{H. BRENNER}

So we are considering the group homomorphism $\varphi: H^{1}(C, \mathcal{F}) \rightarrow H^{1}(C, \mathcal{F})$ and its iterations. Note that $\varphi$ is $p^{v}$-linear, meaning that $\varphi(\lambda c)=\lambda^{p^{v}} \varphi(c)$. In this situation we have an $\mathbb{F}$-vector space decomposition $H^{1}(C, \mathcal{F})=V_{s} \oplus V_{n}$ such that the action of $\varphi$ on $V_{s}$ is bijective and on $V_{n}$ is nilpotent [Mum70, 14, last corollary]. Now a cohomology class $c \in H^{1}(C, \mathcal{T})$ is annihilated by some Frobenius power if and only if the image of $c$ under $\operatorname{proj}_{s} \circ \Phi^{t *}: H^{1}(C, \mathcal{T}) \rightarrow H^{1}(C, \mathcal{F}) \rightarrow V_{s}$ is zero. The dimension of $H^{1}(C, \mathcal{F})$ is bounded by an invariant dependent only on $r$ and $C$, which follows from [Ses82, Lemme 20]. Every application of $\varphi$ annihilates at least one dimension of $V_{n}$, so there is a certain power of $\varphi$ which annihilates $V_{n}$.

Case 3. Now suppose that $\operatorname{deg}(\mathcal{T})<0$. Even in this case it might happen that some Frobenius power of a non-zero element $c \in H^{1}(C, \mathcal{T})$ is zero, but this is rather an exception. First note that the dual sheaf $\mathcal{T}^{\vee}$ has positive degree and is therefore ample, since it is strongly semistable. Consider the extension $0 \rightarrow \mathcal{T} \rightarrow \mathcal{T}^{\prime} \rightarrow \mathcal{O}_{C} \rightarrow 0$ defined by $c \in H^{1}(C, \mathcal{T})$ and consider the dual sequence $0 \rightarrow \mathcal{O}_{C} \rightarrow\left(\mathcal{T}^{\prime}\right)^{\vee} \rightarrow \mathcal{T}^{\vee} \rightarrow 0$ and its Frobenius pull-backs

$$
0 \longrightarrow \mathcal{O}_{C} \longrightarrow \Phi^{t *}\left(\mathcal{T}^{\prime}\right)^{\vee} \longrightarrow \Phi^{t *}\left(\mathcal{T}^{\vee}\right) \longrightarrow 0 .
$$

Now let $k$ be such that

$$
\operatorname{deg}\left(\Phi^{k *}\left(\mathcal{T}^{\prime}\right)^{\vee}\right)=\operatorname{deg}\left(\Phi^{k *}(\mathcal{T})^{\vee}\right)=p^{k} \operatorname{deg}\left(\mathcal{T}^{\vee}\right)>\operatorname{rk}(\mathcal{T})(\operatorname{rk}(\mathcal{T})+1) g
$$

(note that this is satisfied if $p^{k}>\operatorname{rk}(\mathcal{T})(\operatorname{rk}(\mathcal{T})+1) g$ ). Then either $\Phi^{k *}(c)=0$ and $c$ is annihilated by this Frobenius power, or $\Phi^{k *}(c) \neq 0$, the extension remains non-trivial and $\Phi^{k *}\left(\mathcal{T}^{\prime}\right)^{\vee}$ is ample. This follows from [Gie71, Lemma 2.2] and an argument similar to [Gie71, Proposition 2.2]; see [Bre06, Lemma 5.19]. But then the torsor defined by $c$, that is $\mathbb{P}\left(\left(\mathcal{T}^{\prime}\right)^{\vee}\right)-\mathbb{P}\left(\mathcal{T}^{\vee}\right)$, is an affine variety. Therefore $c$ cannot be annihilated by any Frobenius power, because otherwise the pull-back of this torsor would admit a section, contrary to affineness.

Theorem 4.2. Let $C$ denote a smooth projective curve of genus $g$ defined over a finite field $\mathbb{F}$. Fix $r \in \mathbb{N}$ and suppose that $\operatorname{char}(\mathbb{F})=p>4(g-1) r^{3}$. Then there exists a number $b$ such that for every locally free sheaf $\mathcal{S}$ of rank $r$ on $C$ the following holds: if a cohomology class $c \in H^{1}(C, \mathcal{S})$ is annihilated by some Frobenius power, then already $\Phi^{b *}(c)=0$ holds in $H^{1}\left(C, \Phi^{b *}(\mathcal{S})\right)$.

Proof. By Corollary 3.2 there exists a Frobenius power $\Phi^{h}$ such that the Harder-Narasimhan filtration of $\Phi^{h *}(\mathcal{S})$ is strong, say $0 \subset \mathcal{S}_{1} \subset \cdots \subset \mathcal{S}_{t}=\Phi^{h *}(\mathcal{S})$. Let $\mathcal{T}=\mathcal{S}_{j} / \mathcal{S}_{j-1}$ be one of the strongly semistable quotient sheaves in this filtration, so that $\operatorname{rk}(\mathcal{T}) \leqslant r$. By Lemma 4.1 we know that for all these bundles there exists a checking bound for Frobenius annihilation. Let $k$ be such a common bound and set $b=h+r k$.

Now let $c \in H^{1}(C, \mathcal{S})$ denote a cohomology class and suppose that it is annihilated by some Frobenius power. We want to show that already the $b$ th Frobenius power annihilates this class. Let $c_{t}=\Phi^{h *}(c) \in H^{1}\left(C, \Phi^{h *}(\mathcal{S})\right)$ be the pull-back and consider what happens to it under the short exact sequence

$$
0 \longrightarrow \mathcal{S}_{t-1} \longrightarrow \Phi^{h *}(\mathcal{S}) \longrightarrow \Phi^{h *}(\mathcal{S}) / \mathcal{S}_{t-1} \longrightarrow 0 .
$$

Since some Frobenius power of $c_{t}$ is zero, this is also true for its image $c_{t}^{\prime} \in H^{1}\left(C, \Phi^{h *}(\mathcal{S}) / \mathcal{S}_{t-1}\right)$. But then $\Phi^{k *}\left(c_{t}^{\prime}\right)=0$ in $H^{1}\left(C, \Phi^{k *}\left(\Phi^{h *}(\mathcal{S}) / \mathcal{S}_{t-1}\right)\right)$ by Lemma 4.1. Therefore $\Phi^{k *}\left(c_{t}\right)$ is the image of a cohomology class $c_{t-1} \in H^{1}\left(C, \Phi^{k *}\left(\mathcal{S}_{t-1}\right)\right)$.

If $\mathcal{S}_{t} / \mathcal{S}_{t-1}$ has negative degree, then $H^{0}\left(C, \mathcal{S}_{t} / \mathcal{S}_{t-1}\right)=0$ by semistability and $H^{1}\left(C, \mathcal{S}_{t-1}\right) \rightarrow$ $H^{1}\left(C, \mathcal{S}_{t}\right)$ is injective, and this holds for all Frobenius powers. Hence also $c_{t-1}$ is annihilated by some Frobenius power. If however $\operatorname{deg}\left(\mathcal{S}_{t} / \mathcal{S}_{t-1}\right) \geqslant 0$, then $\mu_{\min }\left(\mathcal{S}_{t-1}\right)=\mu\left(\mathcal{S}_{t-1} / \mathcal{S}_{t-2}\right)>0$ and every cohomology class in it is annihilated by some Frobenius power. Application of the induction hypothesis to $\mathcal{S}_{t-1}$ gives the result. 


\section{BOUNDS FOR TEST EXPONENTS}

Remark 4.3. The bound obtained in Theorem 4.2 is hardly suitable for computations. The main problem here is the number $n$ from Lemma 3.1, which bounds the number of semistable sheaves of given rank and degree on a curve over a finite field (this number enters also in the second case in the proof of Lemma 4.1). The dimension of the moduli space of semistable sheaves of rank $r$ and degree $d$ is $r^{2}(g-1)+1$ (see [Ses82, after théorème 18] or [HL97, Corollary 4.5.5]; note that Huybrechts and Lehn give the dimension for a fixed determinant, and this explains the difference of $g$ ).

\section{Frobenius test exponents for ideals in two-dimensional rings}

We now come back to Frobenius test exponents for ideals in two-dimensional rings.

Theorem 5.1. Let $\mathbb{F}$ denote a finite field and let $R$ denote a geometrically normal two-dimensional standard-graded domain over $\mathbb{F}$. Fix $n \in \mathbb{N}$ and suppose that char $(\mathbb{F})=p \geqslant 4(g-1)(n-1)^{3}$, where $g$ denotes the genus of the smooth projective curve $C=\operatorname{Proj} R$. Then there exists a Frobenius test exponent for the class of homogeneous ideals generated by at most $n$ homogeneous elements.

Proof. Suppose first that $I$ is an $R_{+}$-primary ideal. Let $I=\left(f_{1}, \ldots, f_{n}\right)$ and suppose as in the proof of Theorem 1.1 that $f \in I^{\mathrm{F}}$ is homogeneous of degree $m$. Let $\delta(f) \in H^{1}\left(C, \operatorname{Syz}\left(f_{1}, \ldots, f_{n}\right)(m)\right)$. Then $f^{p^{e}} \in I^{\left[p^{e}\right]}$ if and only if $\delta(f)$ is annihilated by the eth Frobenius power. By Theorem 4.2, we know that $\delta(f)$ is annihilated already by the $b$ th Frobenius power, where $b$ is the test bound for locally free sheaves of rank $r=(n-1)$.

Suppose now that $I$ is a homogeneous ideal, but not necessarily $R_{+}$-primary, say $I=\left(h_{1}, \ldots, h_{k}\right)$. Suppose that $f$ is a homogeneous element of degree $m$. Let $x, y$ denote homogeneous parameters in $R$ of degree greater than $m$. If now $f \in I^{\mathrm{F}}$, then also $f \in(I+(x, y))^{\mathrm{F}}$ (which is the Frobenius closure of an $R_{+}$-primary ideal generated by $k+2$ elements), and hence $f^{p^{b}} \in\left(h_{1}^{p^{b}}, \ldots, h_{k}^{p^{b}}, x^{p^{b}}, y^{p^{b}}\right)$ (where $b$ is now the bound for sheaves of rank $k+1$ ). If we write this as a homogeneous equation $f^{p^{b}}=g_{1} h_{1}^{p^{b}}+\cdots+g_{k} h_{k}^{p^{b}}+g_{k+1} x^{p^{b}}+g_{k+2} y^{p^{b}}$ we see that already $f^{p^{b}} \in I^{\left[p^{b}\right]}$.

Remark 5.2. If we delete the condition on the prime number in Theorem 5.1, we still get a positive answer to the weak question of Katzman and Sharp for primary ideals in a two-dimensional geometrically normal standard-graded domain over a finite field. If $I=\left(f_{1}, \ldots, f_{n}\right)$ is the primary ideal, then its syzygy bundle has a strong Harder-Narasimhan filtration on a certain Frobenius pull-back: let us say that the Harder-Narasimhan filtration of the $h$ th pull-back is strong. Now the syzygy bundle of $I^{[q]}=\left(f_{1}^{q}, \ldots, f_{n}^{q}\right)$, where $q=p^{e}$, that is $\operatorname{Syz}\left(f_{1}^{q}, \ldots, f_{n}^{q}\right)$, is just the eth pull-back of $\operatorname{Syz}\left(f_{1}, \ldots, f_{n}\right)$, and therefore the $h$ th pull-back of $\operatorname{Syz}\left(f_{1}^{q}, \ldots, f_{n}^{q}\right)$ has also a strong Harder-Narasimhan filtration. This replaces Corollary 3.2.

\section{Tight closure test ideal exponents}

We recall briefly the notions of tight closure, test ideals and test exponent for tight closure, referring to [Hun98] and [HH00] for details. Let $R$ denote a noetherian commutative ring of positive prime characteristic $p$, and let $R^{\circ}$ denote the complement of the union of the minimal prime ideals. Then the tight closure of an ideal $I$ is defined as the ideal

$$
I^{*}=\left\{f \in R: \exists z \in R^{\circ} \text { such that } z f^{q} \in I^{[q]} \text { for all } q \gg 0\right\} .
$$

An element $z \in R^{\circ}$ is called a test element if for all ideals $I$ and all $f \in I^{*}$ we have $z f^{q} \in I^{[q]}$ for all powers $q=p^{e}$. The test ideal, denoted $\tau$, is the ideal generated by all test elements. It is a non-trivial fact that test elements exist [Hun98, Theorem 3.2] under certain conditions. The situation for Gorenstein local or graded rings is even better: in this case for an arbitrary system of 


\section{H. BRENNER}

parameters we have $\left(x_{1}, \ldots, x_{d}\right): \tau=\left(x_{1}, \ldots, x_{d}\right)^{*}$ and $\tau=\left(x_{1}, \ldots, x_{d}\right):\left(x_{1}, \ldots, x_{d}\right)^{*}$; see [Hun98, Corollaries 4.2 and 4.3]. More specifically, for a noetherian non-negatively graded Gorenstein ring and $p \gg 0$ we have that $\tau=R_{\geqslant a+1}$, where $a$ is the $a$-invariant of $R$. This is the maximal degree $\delta$ such that $\left(H_{R_{+}}^{d}(R)\right)_{\delta} \neq 0$; equivalently, $\mathcal{O}_{C}(a)$ is the dualizing sheaf on $C=\operatorname{Proj} R$ (see [BH98, Proposition 3.6.11]).

Fix a test element $z \in R$, and let $I \subseteq R$ be an ideal. A test exponent for $z$ and $I$ is a prime power $p^{b}$ (or rather its exponent) such that $z f^{p^{b}} \in I^{\left[p^{b}\right]}$ ensures that $f \in I^{*}$ (see [HH00, Definition 2.2], where the definition is given for $R$-modules $N \subseteq M$ ).

Test exponents are important for two reasons. On the one hand, the existence of test exponents is equivalent to the localization of tight closure; see [HH00, Theorem 2.4] for the precise statement. On the other hand, a test exponent gives at once a finite algorithm for the computation of tight closure. Note that, contrary to the case of Frobenius test exponents, already the existence of a test exponent for a single ideal is a problem, not to mention the existence of a uniform bound for test exponents for a reasonable class of ideals [HH00, Discussion 5.3].

Here we will however focus on the following variant of test exponent, which is easier to handle.

Definition 6.1. Let $R$ denote a noetherian ring of positive characteristic $p$ and let $\tau$ denote the test ideal. A test ideal exponent for tight closure (for a certain class of ideals in $R$ ) is a number $b$ such that the following holds: if $z f^{p^{e}} \in I^{\left[p^{e}\right]}$ holds for every $e \leqslant b$ and for every $z \in \tau$, then $f \in I^{*}$.

The existence of a test ideal exponent has the same computational impact on tight closure as the existence of a test exponent for a certain test element. Moreover, its existence implies also that tight closure commutes with localization, at least if all the test elements are locally stable (look at the proof of [HH00, Proposition 2.3]).

We restrict now to the situation of a two-dimensional standard-graded normal Gorenstein domain $R$. We will show that in this case a uniform tight closure test ideal exponent exists for the ideals generated by $n$ homogeneous elements. For a homogeneous element $f$ of degree $m$ and a homogeneous element $z$ the condition that $z f^{q} \in\left(f_{1}, \ldots, f_{n}\right)^{[q]}$ holds translates into $z \Phi^{e *}(\delta(f))=0$ in $H^{1}\left(C, \mathcal{O}_{C}(\operatorname{deg}(z)) \otimes \Phi^{e *}(\mathcal{S})\right)$, where $\mathcal{S}=\operatorname{Syz}\left(f_{1}, \ldots, f_{n}\right)(m), \delta(f) \in H^{1}(C, \mathcal{S})$ and $q=p^{e}$. We will need the following lemma.

Lemma 6.2. Let $C$ denote a smooth projective curve of genus $g$ over a field $\mathbb{F}$. Fix a very ample invertible sheaf $\mathcal{O}_{C}(1)$ and set $\operatorname{deg}(C)=\operatorname{deg}\left(\mathcal{O}_{C}(1)\right)$. Let $u$ be a number such that $u \operatorname{deg}(C)>$ $2 g-2=\operatorname{deg}\left(\omega_{C}\right)$, where $\omega_{C}$ denotes the dualizing sheaf on $C$. Then for every semistable sheaf $\mathcal{T}$ on $C$ of rank $r$ and of degree $\operatorname{deg}(\mathcal{T})<-r(1+(u+1) \operatorname{deg}(C))$ and for every cohomology class $0 \neq c \in H^{1}(C, \mathcal{T})$ there exists a sheaf homomorphism $\varphi: \mathcal{T} \rightarrow \mathcal{O}_{C}(-u) \otimes \omega_{C}$ such that $\varphi(c) \neq 0$ in $H^{1}\left(C, \mathcal{O}_{C}(-u) \otimes \omega_{C}\right)$.

Proof. Suppose that $\mathcal{T}$ satisfies the stated degree condition. Then

$$
\operatorname{deg}\left(\mathcal{T}^{\vee} \otimes \omega_{C} \otimes \mathcal{O}_{C}(-u-1)\right)=-\operatorname{deg}(\mathcal{T})+r(2 g-2)-r(u+1) \operatorname{deg}(Y)>r(2 g-1) .
$$

By [Ses82, Lemme 20] the sheaf $\mathcal{T}^{\vee} \otimes \omega_{C} \otimes \mathcal{O}_{C}(-u-1)$ is therefore generated by its global sections and $H^{1}\left(C, \mathcal{T}^{\vee} \otimes \omega_{C} \otimes \mathcal{O}_{C}(-u-1)\right)=0$. This last property means that $\mathcal{T}^{\vee} \otimes \omega_{C} \otimes \mathcal{O}_{C}(-u)$ is 0-regular in the sense of Castelnuovo and Mumford [HL97, Definition 1.7.1]. Therefore by [HL97, Lemma 1.7.2] we have a surjective map

$$
H^{0}\left(C, \mathcal{T}^{\vee} \otimes \omega_{C} \otimes \mathcal{O}_{C}(-u)\right) \otimes H^{0}\left(C, \mathcal{O}_{C}(n)\right) \longrightarrow H^{0}\left(C, \mathcal{T}^{\vee} \otimes \omega_{C} \otimes \mathcal{O}_{C}(n-u)\right)
$$

for every $n \geqslant 0$. Taking $n=u$ we get a surjective mapping $\varphi: \bigoplus \mathcal{O}_{C}(u) \rightarrow \mathcal{T}^{\vee} \otimes \omega_{C}$ which is also globally surjective. Consider the exact sequence

$$
0 \longrightarrow \mathcal{K} \longrightarrow \bigoplus \mathcal{O}_{C}(u) \stackrel{\varphi}{\longrightarrow} \mathcal{T}^{\vee} \otimes \omega_{C} \longrightarrow 0
$$




\section{BOUNDS FOR TEST EXPONENTS}

This induces the exact sequence

$$
\bigoplus H^{0}\left(C, \mathcal{O}_{C}(u)\right) \stackrel{\varphi}{\longrightarrow} H^{0}\left(C, \mathcal{T}^{\vee} \otimes \omega_{C}\right) \longrightarrow H^{1}(C, \mathcal{K}) \longrightarrow \bigoplus H^{1}\left(C, \mathcal{O}_{C}(u)\right) .
$$

Since $H^{1}\left(C, \mathcal{O}_{C}(u)\right)=H^{0}\left(C, \mathcal{O}_{C}(-u) \otimes \omega_{C}\right)^{\vee}=0$ in view of the assumption on $u$, and since $\varphi$ is surjective on the global sections by construction, we get $H^{1}(C, \mathcal{K})=0$. Now tensor the short exact sequence with $\omega_{C}^{-1}$ and consider the dual sequence

$$
0 \longrightarrow \mathcal{T} \longrightarrow \bigoplus \mathcal{O}(-u) \otimes \omega_{C} \longrightarrow \mathcal{K}^{\vee} \otimes \omega_{C} \longrightarrow 0 .
$$

Since $H^{0}\left(C, \mathcal{K}^{\vee} \otimes \omega_{C}\right)=H^{1}(C, \mathcal{K})^{\vee}=0$, we see that the induced map

$$
H^{1}(C, \mathcal{T}) \longrightarrow \bigoplus H^{1}\left(C, \mathcal{O}_{C}(-u) \otimes \omega_{C}\right)
$$

is injective. Hence for a class $0 \neq c \in H^{1}(C, \mathcal{T})$ at least one component in $H^{1}\left(C, \mathcal{O}_{C}(-u) \otimes \omega_{C}\right)$ is non-zero.

Lemma 6.3. Let $R$ denote a standard-graded two-dimensional geometrically normal Gorenstein domain over a field $\mathbb{F}$ of positive characteristic $p$. Let $C=$ Proj $R$ denote the smooth projective curve of genus $g$ determined by $R$. Set $\operatorname{deg}(C)=\operatorname{deg}\left(\mathcal{O}_{C}(1)\right)$ and let $\omega_{C}=\mathcal{O}_{C}(a)$ be the dualizing sheaf. Let $\tau$ denote the test ideal of $R$. Fix $r \in \mathbb{N}$. Let $k$ be such that $2^{k}>r(1+(a+g+2) \operatorname{deg}(C))$. Then $k$ has the property that for every strongly semistable sheaf $\mathcal{T}$ of rank $r$ and of negative degree the following holds: if for $c \in H^{1}(C, \mathcal{T})$ we have that $z \Phi^{k *}(c)=0$ for all homogeneous $z \in \tau$, then already $\Phi^{k *}(c)=0$.

Proof. Since $\mathcal{T}$ has negative degree, the degree of the $k$ th Frobenius pull-back $\Phi^{k *}(\mathcal{T})$ is

$$
\operatorname{deg}\left(\Phi^{k *}(\mathcal{T})\right)=p^{k} \operatorname{deg}(\mathcal{T}) \leqslant-2^{k}<-r(1+(a+g+1) \operatorname{deg}(C)) .
$$

We want to apply Lemma 6.2 with $u=a+g+1$; note that $(a+g+1) \operatorname{deg}(C)>\operatorname{deg} \mathcal{O}_{C}(a)=$ $2 g-2$. Suppose that $\Phi^{k *}(c) \neq 0$. Then there exists by Lemma 6.2 a sheaf homomorphism $\varphi$ : $\Phi^{k *}(\mathcal{T}) \rightarrow \mathcal{O}_{C}(a-u)=\mathcal{O}_{C}(-g-1)$ such that $0 \neq c^{\prime}=\varphi\left(\Phi^{k *}(c)\right) \in H^{1}\left(C, \mathcal{O}_{C}(-g-1)\right)$. Since $z \Phi^{k *}(c)=0$ for all $z \in \tau$, this holds also for $c^{\prime}$. This means that we have a cohomology class $c^{\prime} \in$ $H^{1}(C, \mathcal{O}(-g-1)) \cong\left(H_{R_{+}}^{2}(R)\right)_{-g-1}$ of negative degree $-g-1$ which is annihilated by the test ideal. By [Hun98, Corollary 4.2(1)] we have that $0^{*}=\left\{c \in H_{R_{+}}^{2}(R): \tau c=0\right\}$ and hence $c^{\prime} \in 0^{*}$. This means that the torsor defined by $c^{\prime}$ is not affine, but since $\operatorname{deg}\left(\mathcal{O}_{C}(-g-1)\right)=-(g+1) \operatorname{deg}(C)<$ $-2 g$, results of Gieseker [Gie71, Lemma 2.2 and Proposition 2.2] imply that $c^{\prime}=0$, a contradiction.

Theorem 6.4. Let $R$ denote a standard-graded two-dimensional geometrically normal Gorenstein domain over a finite field $\mathbb{F}$ of positive characteristic $p$. Fix $n \in \mathbb{N}$ and suppose that $p \geqslant 4(g-1)(n-1)^{3}$, where $g$ denotes the genus of $C=\operatorname{Proj} R$. Then there exists a test ideal exponent for the class of homogeneous ideals generated by at most $n$ elements.

Proof. We have to show that there exists a $b$ such that for every homogeneous ideal $I=\left(f_{1}, \ldots, f_{n}\right)$ the following holds: if for an element $f \in R$ we have $z f^{q} \in I^{[q]}$ for all $z \in \tau$ and all $q=p^{e}, e \leqslant b$, then already $f \in I^{*}$.

Again we may reduce to the primary case as in the proof of Theorem 5.1. Set $b=h+(n-1) k$, where $h$ is the bound from Corollary 3.2 for locally free sheaves of rank $n-1$ on $C$ and where $k$ is a common bound from Lemma 6.3 for the strongly semistable sheaves of ranks $r \leqslant n-1$. We can also reduce to the homogeneous case, so let $f$ denote a homogeneous element of degree $m$. Suppose that $z f^{q} \in I^{[q]}$ for all $z \in \tau$ and all $q=p^{e}, e \leqslant b$; we have to show that this is then true for every power 


\section{H. BRENNER}

of $p$ (and at least for one element $\left.z \in R^{\circ}\right)$. Let $\mathcal{S}=\operatorname{Syz}\left(f_{1}, \ldots, f_{n}\right)(m)$ denote the syzygy bundle of rank $n-1$ on the smooth projective curve $C=\operatorname{Proj} R$ and let $c=\delta(f) \in H^{1}\left(C, \operatorname{Syz}\left(f_{1}, \ldots, f_{n}\right)(m)\right)$.

Using Corollary 3.2 we get the strong Harder-Narasimhan filtration $\mathcal{S}_{0} \subset \cdots \subset \mathcal{S}_{t}=\Phi^{h *}(\mathcal{S})$; consider $c_{t}=\Phi^{h *}(c) \in H^{1}\left(C, \Phi^{h *}(\mathcal{S})\right)$. Then we consider the image $c_{t}^{\prime}$ of $c_{t}$ in $H^{1}\left(C, \mathcal{S}_{t} / \mathcal{S}_{t-1}\right)$. If $\mathcal{T}=\mathcal{S}_{t} / \mathcal{S}_{t-1}$ has negative degree, then by Lemma 6.3 we have that $\Phi^{k *}\left(c_{t}^{\prime}\right)=0$, since it is annihilated by the test ideal. In this case $\Phi^{k *}\left(c_{t}\right)$ is the image of a class $c_{t-1} \in H^{1}\left(C, \Phi^{k *}\left(\mathcal{S}_{t-1}\right)\right)$. Since $H^{0}\left(C, \Phi^{e *}(\mathcal{T})\right)=0$, we have $H^{1}\left(C, \Phi^{e *}\left(\mathcal{S}_{t-1}\right)\right) \subseteq H^{1}\left(C, \Phi^{e *}\left(\mathcal{S}_{t}\right)\right)$ for all $e$ and it follows from our assumption that $z \Phi^{e *}\left(c_{t-1}\right)=0$ for $e \leqslant(n-2) k$ and all $z \in \tau$. If however $\mathcal{T}$ has non-negative degree, then the minimal slope of $\mathcal{S}_{t}=\Phi^{h *}(\mathcal{S})$ is non-negative and then $z \Phi^{e *}(c)=0$ for $z \in R_{\geqslant a+1}$ and all $e$ anyway (even in small characteristics, where $\tau$ may be different from $R_{\geqslant a+1}$ ). In this way we use induction along the strong Harder-Narasimhan filtration.

Remark 6.5. The problem with test exponents for a given fixed test element $z$ is that even in the case of a parameter ideal (which corresponds to a cohomology class in an invertible sheaf) it is not clear how to bound the test exponent [HH00, Discussion 5.1].

Corollary 6.6. Let $\mathbb{F}$ denote a field of positive characteristic $p$ and let $G \in \mathbb{F}[x, y, z]$ denote a cubic polynomial such that $C=\operatorname{Proj} R$, where $R=\mathbb{F}[x, y, z] /(G)$, is an elliptic curve. Let $e$ be such that $p^{e}>7(n-1)$. Then $e$ is a test ideal exponent for the set of all homogeneous $R_{+}$-primary ideals generated by $n$ homogeneous elements.

Proof. Since $g(C)=1, \operatorname{deg}(C)=3$ and $\omega_{C}=\mathcal{O}_{C}$, Lemma 6.2 applied with $u=1$ shows that for every semistable sheaf $\mathcal{T}$ on $C$ of rank $r$ and of degree $\operatorname{deg}(\mathcal{T})<-7 r$ and a cohomology class $c \in H^{1}(C, \mathcal{T})$ there exists a sheaf homomorphism $\varphi: \mathcal{T} \rightarrow \mathcal{O}_{C}(-1)$ such that $\varphi(c) \neq 0$ in $H^{1}\left(C, \mathcal{O}_{C}(-1)\right)$.

Let $q=p^{e}>7(n-1)$. Let $I=\left(f_{1}, \ldots, f_{n}\right)$ denote an $R_{+}$-primary ideal generated by $n$ homogeneous elements and let $f \in R$ denote an element of degree $m$. The test ideal of $R$ is the maximal ideal $\tau=(x, y, z)$. So suppose that $w f^{q} \in\left(f_{1}^{q}, \ldots, f_{n}^{q}\right)=I^{[q]}$ for $w=x, y, z$. We have to show that $f \in I^{*}$. Let $\operatorname{Syz}\left(f_{1}, \ldots, f_{n}\right)(m) \cong \mathcal{S}_{1} \oplus \cdots \oplus \mathcal{S}_{k}$ denote the decomposition of the syzygy bundle on $C$ into indecomposable sheaves and let

$$
\delta(f)=c=\left(c_{1}, \ldots, c_{k}\right) \in H^{1}\left(C, \operatorname{Syz}\left(f_{1}, \ldots, f_{n}\right)(m)\right)=\bigoplus_{j=1}^{k} H^{1}\left(C, \mathcal{S}_{j}\right)
$$

denote the components of the cohomology class. Assume that $f \notin I^{*}$. By [Bre05, Corollary 4.1] there exists $j, 1 \leqslant j \leqslant k$, such that $\mathcal{S}_{j}$ has negative degree and $c_{j} \neq 0$. Consider $\Phi^{e *}(c) \in H^{1}\left(C, \Phi^{e *}\left(\mathcal{S}_{j}\right)\right)$. Recall that indecomposable sheaves on an elliptic curve are strongly semistable. Furthermore note that $\Phi^{e *}(c) \neq 0$, otherwise $f$ would belong to the tight closure. Since $\operatorname{deg}\left(\Phi^{e *}\left(\mathcal{S}_{j}\right)\right) \leqslant p^{e}<-7(n-1)$ $\leqslant-7 \operatorname{rk}\left(\mathcal{S}_{j}\right)$, there exists a morphism $\varphi: \Phi^{e *}\left(\mathcal{S}_{j}\right) \rightarrow \mathcal{O}_{C}(-1)$ such that $0 \neq c^{\prime}=\varphi\left(\Phi^{e *}\left(c_{j}\right)\right) \in$ $H^{1}\left(C, \mathcal{O}_{C}(-1)\right)$. By assumption the class $\Phi^{e *}\left(c_{j}\right)$ is annihilated by $(x, y, z)$, and this property passes over to $c^{\prime}$. But for $0 \neq c^{\prime} \in H^{1}\left(C, \mathcal{O}_{C}(-1)\right)$ there exists, by Serre duality, a homomorphism $\theta$ : $\mathcal{O}_{C}(-1) \rightarrow \mathcal{O}_{C}=\omega_{C}$ such that $\theta\left(c^{\prime}\right) \neq 0$. However, $\theta$ is given by a linear form, and so we have a contradiction.

\section{ACKNOWLEDGEMENTS}

I would like to thank M. Hochster, M. Katzman, R. Sharp, K. Smith, the referee and in particular R. Lazarsfeld for their interest and for useful comments. 


\section{BOUNDS FOR TEST EXPONENTS}

\section{REFERENCES}

Ati57 M. F. Atiyah, Vector bundles over an elliptic curve, Proc. London Math. Soc. (3) 7 (1957), 414-452.

Bre05 H. Brenner, Tight closure and plus closure for cones over elliptic curves, Nagoya Math. J. 177 (2005), 31-45.

Bre06 H. Brenner, Tight closure and vector bundles, Winter School on Commutative Algebra and Applications, Barcelona, 2006.

BH98 W. Bruns and J. Herzog, Cohen-Macaulay rings, revised edition (Cambridge University Press, 1998).

Gie71 D. Gieseker, p-ample bundles and their Chern classes, Nagoya Math. J. 43 (1971), 91-116.

HH00 M. Hochster and C. Huneke, Localization and test exponents for tight closure, Michigan Math. J. 48 (2000), 305-329.

Hun98 C. Huneke, Tight closure, parameter ideals, and geometry, in Six lectures on commutative algebra (Birkhäuser, Basel, 1998).

HL97 D. Huybrechts and M. Lehn, The geometry of moduli spaces of sheaves (Vieweg, Braunschweig, 1997).

KS04 M. Katzman and R. Y. Sharp, Uniform behaviour of the Frobenius closures of ideals generated by regular sequences, J. Algebra 295 (2004), 231-246.

LS77 H. Lange and U. Stuhler, Vektorbündel auf Kurven und Darstellungen der algebraischen Fundamentalgruppe, Math. Z. 156 (1977), 73-83.

Lan04 A. Langer, Semistable sheaves in positive characteristic, Ann. of Math. (2) 159 (2004), 251-276.

Mum70 D. Mumford, Abelian varieties (Oxford University Press, 1970).

Oda71 T. Oda, Vector bundles on an elliptic curve, Nagoya Math. J. 43 (1971), 41-72.

Ses82 C. S. Seshadri, Fibrés vectoriels sur les courbes algébriques, Asterisque 96 (1982).

Tri04 V. Trivedi, Hilbert-Kunz multiplicity and reduction mod p, Preprint (2004), arXiv:math.AC/0407513.

Holger Brenner h.brenner@sheffield.ac.uk

Department of Pure Mathematics, University of Sheffield, Hicks Building, Hounsfield Road, Sheffield S3 7RH, UK 